\title{
OPTICAL NETWORK UNIT BASED ON A BIDIRECTIONAL REFLECTIVE SEMICONDUCTOR OPTICAL AMPLIFIER
}

\author{
Josep Prat ${ }^{1,2}$, Cristina Arellano ${ }^{1,3}$, Victor Polo ${ }^{1,4}$, Carlos Bock $^{1,5}$ \\ ${ }^{1}$ Dep.of Signal Theory and Communications, Universitat Politècnica de Catalunya, \\ C/ Jordi Girona D-4, 08034 Barcelona \\ ${ }^{2}$ jprat@tsc.upc.es \\ ${ }^{3}$ cristina.arellano@tsc.upc.es \\ ${ }^{4}$ polo@tsc.upc.es \\ sbock@tsc.upc.es
}

\begin{abstract}
An optical access network transceiver based on a reflective semiconductor optical amplifier (RSOA) operating as modulator and photodetector is demonstrated. The system shows proper operation at $1.25 \mathrm{Gbit} / \mathrm{s}$ to $30 \mathrm{~km}$ reach.
\end{abstract}

Keywords: Access networks, Fiber-to-the-home, Optical modulation, Optical signal detection, Reflective semiconductor optical amplifier.

\section{INTRODUCTION}

Fiber-to-the-Home technology is one of the main research objectives in the "Broadband for all" concept that encourages the development of optical access infrastructure [1]. In order to fulfill this concept, cost effective solutions must be developed to be able to offer broadband connections to end users at a reasonable cost. A key element in access networks is the Optical Network Unit (ONU) of the Customer Premises Equipment (CPE). This has a direct impact on the cost per customer and in general on the overall cost of the access network. Therefore, simple ONUs needs to be designed. Also, a desirable characteristic of the outside plant of an access network is the use of one single fiber for both upstream and downstream transmission to reduce network size and connection complexity [2]. 
Some ONU designs that avoid the local generation of light have been lately demonstrated that fulfill the above simplicity criteria. The laser and its stabilization electronics are avoided, and being all ONUs equal while maintaining the necessary transparency for the WDM operation of the network. This is accomplished by using different modulating formats for upstream and downlink transmission or some amplitude signal regeneration, and advanced devices: Phase-Shift Keying/Intensity Modulation [3], Electro-Absortion Transceiver for signal remodulation [4], polarization rotation remodulator [5] and IM remodulation using semiconductor optical amplifiers [6].

We here demonstrate the use of a Reflective Semiconductor Optical Amplifier (RSOA) as a potential candidate for an electro-optic transceiver at the ONU, operating in burst mode. The advantages of this device are inherent optical amplification of the incoming signal and the use of a single fiber in the outside plant. Incoming light can be modulated by the RSOA injection current carrying the upstream user data [7]. The RSOA can also act as a photodetector by sensing the voltage variation of the electrode [8]. In this paper we integrate both functionalities, detection and modulation, in a FTTH network with single fiber access.

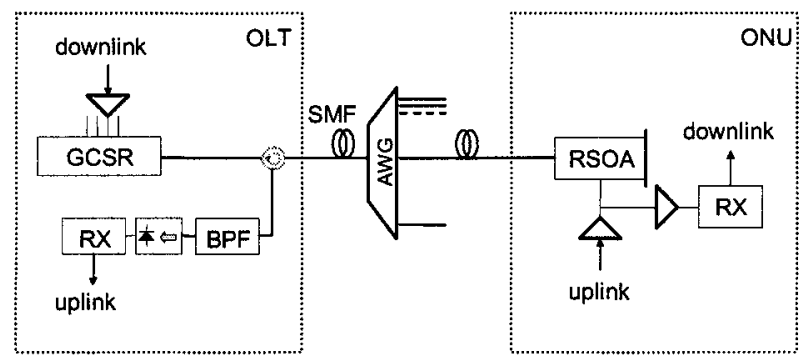

Figure 1. Schematic of the FTTH Network

\section{SYSTEM SCHEME}

Figure 1 depicts the network topology and the design of the proposed Optical Layer Termination (OLT) and ONU. A Grating-assisted codirectional Coupler with rear Sampled Reflector (GCSR) is used at the OLT as a light source. Downstream data is modulated up to $1.25 \mathrm{Gbit} / \mathrm{s}$ using a $\mathrm{LiNbO}_{3}$ modulator, and sent through an optical circulator that will play its role when receiving upstream data. A 1x8 AWG routes optical signals depending on input wavelength to the desired ONU. The ONU is built just by one RSOA, which acts as modulator and photodetector. 
Electrically, an interface circuit for the RSOA has been designed to separate the received downstream signal and the ONU upstream data, combining a low noise preamplifier and a laser driver both connected at the current electrode. Two electrical switches are used to activate receive or transmit mode, and avoid the saturation of the preamplifier.

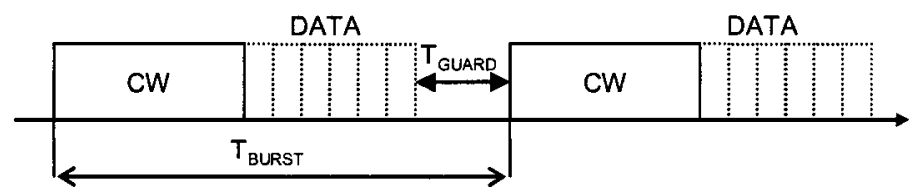

Figure 2. Downlink Burst

Downstream data and upstream carrier are sent from the OLT time multiplexed in a single burst. The first burst section is downstream data and then, after a guard band, optical carrier is sent for upstream modulation purposes. Once upstream data is modulated it is sent back to the OLT; there, the optical circulator routes the incoming signal to the detection branch and isolates the laser source. The data source and receiver are synchronized in burst mode to the corresponding packet period.

\section{EXPERIMENTAL SETUP}

The setup that has been implemented to demonstrate the feasibility of the transmission is very similar to the proposed network topology.

Regarding the RSOA optical specifications, the device has chip length of $500 \mu \mathrm{m}$, reflective facet with $90 \%$ refection and front facet reflecting less than $0.1 \%$. RSOA optical gain has been adjusted to $10 \mathrm{~dB}$ injecting $65 \mathrm{~mA}$ of current. This is the current that has been used during testing because offered enough gain, modulation efficiency, detection sensitivity and enough tolerance against reflections.

Tests have been performed using two separate fibers for upstream and downstream and one single fiber for both directions. Also, detection and modulation tests have been performed. 


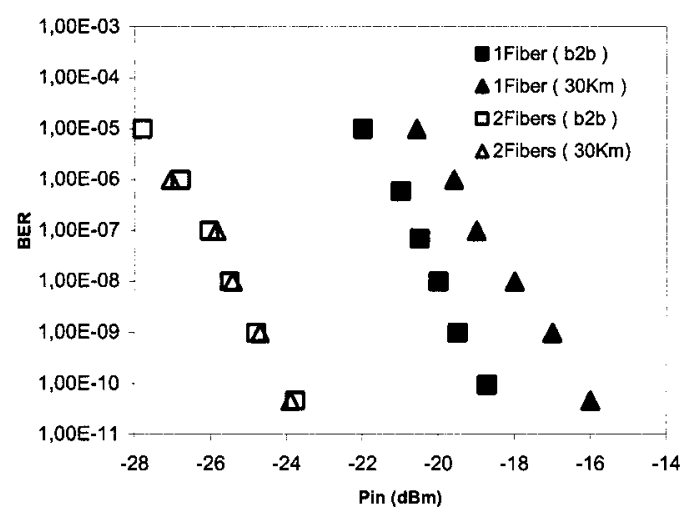

Figure 3. RSOA acting as modulator (Uplink)

Figure 3 presents the results obtained using the RSOA as a modulator. The -25 $\mathrm{dBm}$ sensitivity (at a BER of $10^{-9}$ ) obtained for two different fibers are almost identical in both back-to-back and using $30 \mathrm{~km} \mathrm{SMF}$. The reason is that fiber dispersion is negligible at $1.25 \mathrm{Gbit} / \mathrm{s}$. When the system becomes bidirectional, that is to say, in a single fiber scenario, a $5 \mathrm{~dB}$ degradation of sensitivity has been observed. And also there is a $2 \mathrm{~dB}$ of penalty when SMF fiber is introduced, due to the rayleigh backscattering effect which is one of the most important signal perturbations when transmitting using a single fiber on both directions [9]. With reference to the difference in sensitivity when using one or two fibers, it can be deduced that it is caused by reflections in connectors because of isolation between the circulator ports is not perfect despite APC connectors have been used to minimize reflections.

RSOA detection capabilities have been tested by modulating a $1.25 \mathrm{Gbit} / \mathrm{s}$ data signal using a $\mathrm{LiNbO}_{3}$ modulator and a GCSR laser source located at the OLT. We observe that the RSOA needs a higher optical power (about $-19 \mathrm{dBm}$ ) to be able to produce the detectable variation of voltage. This is because of the certain level of saturation or carrier density reduction in marks. The results that have been obtained in both modulation and detection mode are summarized in Figure 4.

It can be seen that downstream signal is not severely degraded when transmitting using a single fiber along $30 \mathrm{~km}$, though a $2 \mathrm{~dB}$ penalty in sensitivity was measured when the RSOA was working as modulator, in the uplink. The explanation to this difference is that upstream carrier is sent from the OLT and needs to be transmitted twice along the fiber, being more affected by rayleigh scattering than the downstream signal, which just travels in one direction along the fiber; also, the upstream optical power is lower than the downstream, producing a 
lower interference.

We have also tested several optical carriers and have found that when using wide spectrum light sources, rayleigh scattering is less critical than with narrower sources, confirming that backscattering interference is a coherent process.

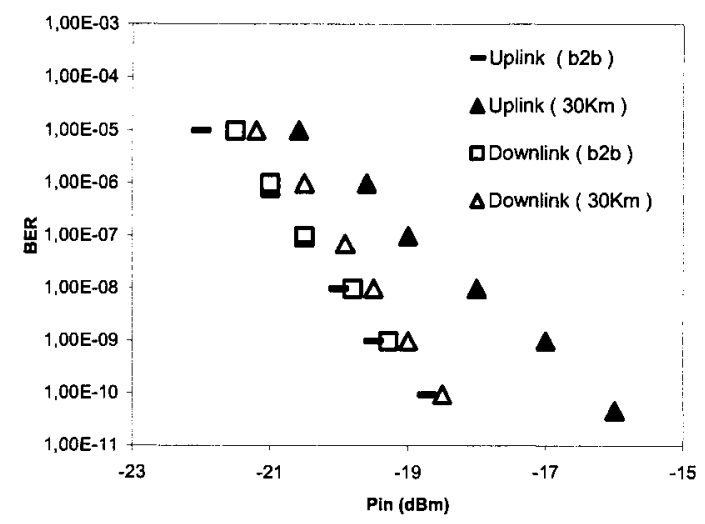

Figure 4. Downlink / Uplink BER comparison

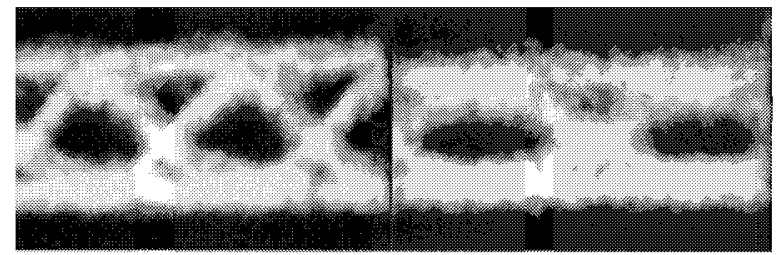

(a)

(b)

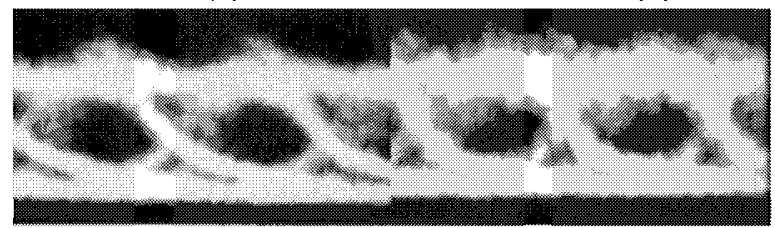

(c)

(d)

Figure 5. RSOA detection/modulation output eye diagrams: (a) detection (downlink) backto-back, (b)detection $30 \mathrm{~km} \mathrm{SMF,} \mathrm{(c)} \mathrm{modulation} \mathrm{(uplink)} \mathrm{back-to-back,} \mathrm{(d)modulation} 30$ km SMF. 


\section{CONCLUSIONS}

A RSOA as Optical Network Unit has been presented as a potential cost effective solution for a FTTH passive optical platform with full-duplex operation along single fiber outside plant.

Modulation and photo detection tests have been implemented in order to demonstrate that this architecture is feasible. Results show that bidirectional communication using one single fiber is possible but rayleigh scattering interference and RSOA sensibility limitations.

\section{ACKNOWLEDGMENTS}

This work was supported by the Spanish MCYT project TIC2002-00053.

\section{REFERENCES}

[1] A. Houghton, "Research in the IST programme: Broadband for all", ECOC 2003 Rimini, Mo3.1.1.

[2] J. Prat, P. E. Balaguer, J.M. Gené, O. Díaz, S. Figuerola, Fiber-to-the-Home Technologies, Kluwer Ac. Publishers, ISBN 1-4020-7136-1.

[3] W. Hung, C-K Chan, L-K Chen and C. Lin, "System characterization of a robust remodulation scheme with DPSK downstream traffic in a WDM access network", Proc. ECOC 2003 Rimini, We3.4.5.

[4] M. Schneider, T. Reimann, A. Stöhr, S. Neumann, F.-J. Tegude, D. Jäger, "Monolithically Integrated Optoelectronic Circuits using HBT, EAM, and TEAT", Proc. MWP 2002, Awaji, Japan, 2002, pp. 349-352, ISBN 4-88552-187-4 C3055.

[5] T. Koonen, K. Steenbergen, F. Janssen, J. Wellen, "Flexibly Reconfigurable FiberWireless Network using Wavelength Routing Techniques: The ACTS Project AC349 PRISMA", Photonic Network Communications, July 2001, pp. 297-306.

[6] H. Takesue and T. Sugie, "Data rewrite of wavelength channel using saturated SOA modulator for WDM Metro/Access networks with centralized light sources", Proc. ECOC 2002 Copenhagen, 8.5.6.

[7] N. Buldawoo et al., "A Semiconductor Laser Amplifier-Reflector for the future FTTH Applications", ECOC 1997 Edinburgh, p196 - 199.

[8] N. Buldawoo et al., "Transmission Experiment using a laser amplifier-reflector for DWDM access network, ECOC 1998 Madrid, pp.273-274.

[9] P. Gysel and R. K. Staubli, "Statistical properties of Rayleigh backscattering in singlemode fibers", J. Lightwave Technol., vol. 8, pp.561-567. 